\title{
Preoperative imaging of the inferior alveolar nerve canal by cone-beam computed tomography and 1-year neurosensory recovery following mandibular setback through bilateral sagittal split ramus osteotomy: a randomized clinical trial
}

\author{
Ali Hassani', Vahid Rakhshan², Mohammad Hassani ${ }^{3}$, Hamidreza Mahaseni Aghdam ${ }^{1}$ \\ ${ }^{\prime}$ Department of Oral and Maxillofacial Surgery and Implant Research Center, Islamic Azad University, Tehran Dental Branch, \\ ${ }^{2}$ Department of Dental Anatomy, Dental School, Islamic Azad University, \\ ${ }^{3}$ Craniomaxillofacial Research Center, Islamic Azad University, Tehran Dental Branch, Tehran, Iran
}

\begin{abstract}
J Korean Assoc Oral Maxillofac Surg 2020;46:41-48)
Objectives: One of the most common complications of bilateral sagittal split ramus osteotomy (BSSRO) is neurosensory impairment of the inferior alveolar nerve (IAN). Accurate preoperative determination of the position of the IAN canal within the mandible using cone-beam computed tomography (CBCT) is recommended to prevent IAN dysfunction during BSSRO and facilitate neurosensory improvement after BSSRO.

Materials and Methods: This randomized clinical trial consisted of 86 surgical sites in 43 patients ( 30 females and 13 males), including 21 cases (42 sides) and 22 controls (44 sides). Panoramic and lateral cephalographs were obtained from all patients. In the experimental group, CBCT was also performed from both sides of the ramus and mandibular body. Neurosensory function of the IAN was subjectively assessed using a 5-point scale preoperatively and 7 days, 1 month, 3 months, 6 months, and 12 months post-surgery. Data were analyzed using Fisher's test, Spearman's test, t-test, linear mixed-model regression, and repeated-measures $\operatorname{ANCOVA}(\alpha=0.05,0.01)$.

Results: Mean sensory scores in the control group were 1.57, 2.61, 3.34, 3.73, and 4.20 over one year and were 1.69, 3.00, 3.60, 4.19, and 4.48 in the CBCT group. Significant effects were detected for CBCT intervention $(P=0.002)$ and jaw side $(P=0.003)$ but not for age $(P=0.617)$ or displacement extent $(P=0.122)$.

Conclusion: Preoperative use of CBCT may help surgeons to practice more conservative surgery. Neurosensory deficits might heal faster on the right side.
\end{abstract}

Key words: Cone-beam computed tomography, Randomized clinical trial

[paper submitted 2019. 6. 6 / revised 2019. 7. 25 / accepted 2019. 8. 17]

\section{Introduction}

One of the most common complications after bilateral sagittal split ramus osteotomy (BSSRO) is neurosensory impairment of the inferior alveolar nerve (IAN) following injury to the nerve bundle during surgery. Such injuries may occur due

\section{Hamidreza Mahaseni Aghdam}

Department of Oral and Maxillofacial Surgery, Buali Hospital, Islamic Azad University of Medical Sciences, Tehran 1711734365, Iran

TEL: +98-9122123906 FAX: +98-2133786182

E-mail:hamidmahaseni@gmail.com

ORCID: https://orcid.org/0000-0002-9811-4254

(c) This is an open-access article distributed under the terms of the Creative Commons Attribution Non-Commercial License (http://creativecommons.org/ licenses/by-nc/4.0/), which permits unrestricted non-commercial use, distribution, and reproduction in any medium, provided the original work is properly cited. Copyright (O) 2020 The Korean Association of Oral and Maxillofacial Surgeons. All rights reserved. to the variable anatomy of the inferior alveolar canal and buccolingual and its vertical orientation ${ }^{1-3}$. This is an important concern in mandibular osteotomy, since patients may experience variable degrees of neurosensory dysfunction for long periods of time; in some cases, dysfunction is permanent ${ }^{1}$. The incidence of IAN dysfunction is reported to range between $13 \%-100 \%$ immediately after surgery to $0 \%-85 \%$ after one year ${ }^{4}$. Surgeons aim to decrease the incidence of IAN dysfunction by improving surgical techniques and performing preoperative imaging such as panoramic views to avoid nerve bundling. However, if they fail, injury to the nerve can lead to complications such as drooling, lip biting, and thermal injury as well as patient dissatisfaction ${ }^{3,5-7}$.

Therefore, preoperative radiographic examinations are of significant clinical value ${ }^{8}$. Although panoramic and lateral 
cephalography is the main method used, it is limited by inconsistencies in magnification of different parts of the image and its inability to exhibit all three-dimensions ${ }^{8-11}$. On the other hand, cone-beam computed tomography (CBCT) can illustrate buccolingual depth without distortion and seems excellent for localizing the inferior alveolar canal (IAC) and adjacent structures ${ }^{8,10,12-14}$. Therefore, accurately determining the position of the IAN canal within the mandible with CBCT before surgery may reduce IAN dysfunction after BSSRO. Despite its clinical relevance, the impact of CBCT combined with routine imaging compared to routine imaging alone on post-surgical sensory disturbances has been examined in only one preliminary study ${ }^{2}$. Therefore, the purpose of this study was to evaluate the effects of preoperative imaging of the IAN canal using CBCT with routine imaging versus routine imaging alone (panoramic and lateral cephalography) on sensory disturbances perceived during the first year after surgery. The null hypotheses were that there would be no differences between groups, between sexes, between left and right sides, over time, or across ages.

\section{Materials and Methods}

This parallel randomized clinical trial comprised 43 patients (30 females and 13 males) with skeletal class III deformity needing mandibular setback surgery with or without simultaneous maxillary surgery who were treated at Buali Hospital in Tehran, Iran during 2014 to 2016. We included patients who were candidates for BSSRO due to mandibular setback less than $7 \mathrm{~mm}$ and excluded patients who had jaw asymmetry, pathologic conditions of the IAN, previous surgery in that region, any neurosensory dysfunction of the IAN, required genioplasty, and had medical conditions with associated neuropathy before surgery. We also excluded patients who were randomly assigned to the CBCT group but who did not consent to undergo CBCT imaging, although they received routine treatment. The protocol ethics were approved by the Islamic Azad University's Board of Ethics and Research according to the Helsinki declaration (No. 08-21214), and informed consent was obtained from each patient. The trial was reviewed by and approved/registered at an international institute before beginning the study (RCT code: IRCT2014052217798N1).

\section{Randomization}

The patients were randomized sequentially to either the control or experimental group based on order of treatment at the clinic (the first patient being randomly assigned to the experimental group). Patients with odd and even numbers were assigned to the experimental and control groups, respectively. Since the order of patients was random and not previously known, this systematic sampling was considered random. The surgeon and patients were not blinded to grouping, but the operator who collected the information was blinded. However, the study is not considered a blind study.

\section{Intervention}

Plain radiographs, including panoramic radiographs and lateral cephalographs, were performed for all patients in both groups. In the experimental group, we performed CBCT (Newtom VG-Quantitative Radiology, Verona, Italy) from both sides of the ramus and mandibular body through the region of the second molar with $2 \mathrm{~mm}$ interval cuts. The distance between the mandibular foramen and sigmoid notch was measured. The distance between the cortical border of the IAN canal and the lateral cortex of the mandible, the thickness of the lateral cortex of the mandible, and the distance between the canal and inferior border of the mandible in the region of the second molar were measured and documented on the CBCT. The regions of the canal near the cortex were documented to avoid nerve bundle injury in these regions.(Fig. 1)

\section{Surgical procedure}

All surgeries were performed by one right-handed, experienced surgeon. The surgical method applied for all patients was BSSRO with the Obwegeser-Dal Pont technique and Hunsuck modification ${ }^{15}$. After incision and during dissection of the medial side of the ramus, attention was paid to the site of the mandibular foramen. After identification of the sigmoid notch, the site of horizontal osteotomy of the medial was determined half-way between the sigmoid notch and mandibular foramen. The medial soft tissues were retracted, and the osteotomy was performed with a Lindemann bur. Vertical osteotomy of the buccal cortex was performed in the region of the second molar to the medullary bone, and crestal osteotomy of the anterior border of the ramus was completed to connect the lingual and buccal osteotomies. Bone splitting was achieved with a chisel in close contact to the inner side of the buccal cortex as it advanced forward. Splitting of cortices was accompanied by searching for and preserving 

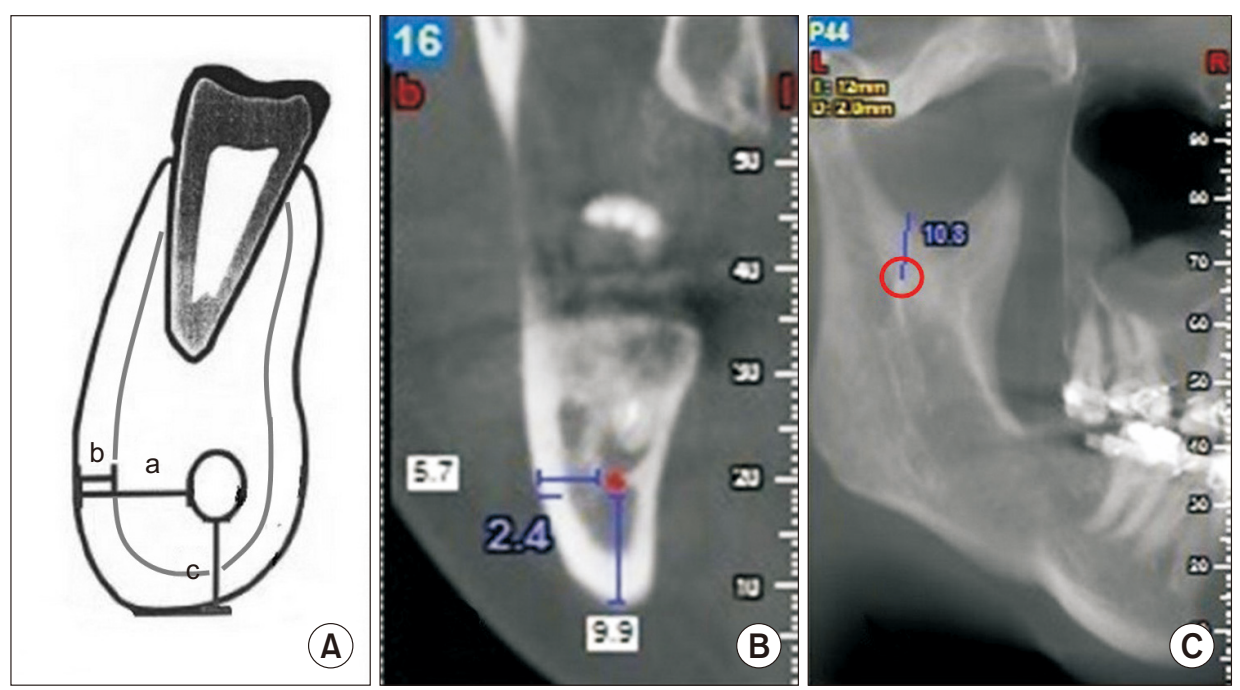

Fig. 1. A. Schematic view of the mandibular body. a: distance between inferior alveolar nerve (IAN) canal and buccal cortex of the mandible, b: thickness of the buccal cortex, c: distance between IAN canal in inferior border. B. Measurements of cone-beam computed tomography. C. Distance between the mandibular foramen (at the center of the red circle) and sigmoid notch. The mandibular foramen was located on images as the starting point of the mandibular canal.

Ali Hassani et al: Preoperative imaging of the inferior alveolar nerve canal by cone-beam computed tomography and 1-year neurosensory recovery following mandibular setback through bilateral sagittal split ramus osteotomy: a randomized clinical trial. J Korean Assoc Oral Maxillofac Surg 2020

the neurovascular bundle. After splitting, any irregularity in the interior surface of the two proximal and distal segments was removed by a round bur. After mobilization, the distal segment was repositioned in occlusion, and a maxillomandibular fixation wire was placed. The proximal segment was repositioned to correct the relationship with the glenoid fossa; in 62 hemimandibles, rigid fixation was achieved with two bicortical screws while paying attention to protect the neuromuscular bundle from injury during screw placement. In 18 hemimandibles, this procedure was performed using one screw. In two hemimandibles, there was no fixation; in two others, three screws were installed, and plates were used for fixation in two others. Dexamethasone $8 \mathrm{mg}$ was injected in all patients before surgery and every 8 hours after surgery for two days, with the dose tapered to $4 \mathrm{mg}$ every 12 hours on the third day after surgery and discontinued the day after that.

When the surgeon predicted IAN damage based on CBCT, the following precautions were taken during surgery. The patient was notified that the risk of IAN damage is high, and that there might be neurosensory disturbances after the surgery. In some cases, it was necessary to change the location of the vertical cut. In areas where the nerve was very close to the lateral cortex, fine osteotomes and splitting methods were used to bypass the danger zone. The exact top-bottom location of the nerve at the lateral side of the mandibular trunk was determined, and in cases of proximity to the lateral cortex, the depth of bone removal was reduced.

\section{Neurosensory evaluation}

Neurosensory functional evaluation of IAN was determined subjectively using a 5-point Likert scale questionnaire following Westermark et al. ${ }^{16}$. The patients were asked to rate the numbness of the right and left sides of the lip and chin areas. On this scale, scores of 1 to 5 were indicative of complete numbness, almost near numbness, some sensation, almost near normal sensation, and normal sensation, respectively. This questionnaire was administered preoperatively and 7 days, 1 month, 3 months, 6 months, and 12 months post-surgery. All preoperative sensory measurements indicated health in all patients (i.e., all scores were 5).

\section{Statistical analysis}

The sample size was pre-determined based on a 6-month pilot study of 10 patients. We determined that $40 \times 2$ patients were necessary to obtain a test power greater than $80 \%$. The pilot sample was not included in the study.

Descriptive statistics and 95\% confidence interval were calculated for all measurements. The extents of mandibular setback were compared between the two groups using an independent-samples t-test. Groups were compared in terms of sex and age using Fisher's exact test and independentsamples t-test, respectively. The Spearman correlation coefficient was used to evaluate correlations between neurosen- 
sory scores on left and right sides and between neurosensory scores and extent of mandibular setback. Independentsamples t-tests were used to compare neurosensory function of control and cohort groups for each of the 5 time points. A paired t-test was used to compare the neurosensory function of left versus right sides at each time point.

The whole dataset was analyzed once using a mixed-effects linear model, discarding the effect of time. Then, the data were divided into two datasets pertaining to the left and right sides, and each side was analyzed separately using repeatedmeasures ANCOVA while accounting for the effect of time but discarding the right/left sides.

A mixed-effects linear model was used to assess the effects of jaw side (left or right), the intervention, displacement extent, and patient age on overall neurosensory function. In each of the left or right subgroups, repeated-measures ANCOVA was used to analyze the effects of time, setback extent, and radiography method (control/experimental) as the intervention on neurosensory function. We conducted analyses using IBM SPSS Statistics (ver. 23; IBM, Armonk, NY, USA). The level of significance was set at 0.05 for all tests, except the two t-tests used for comparisons of neurosensory scores between groups and sides at each time point. For these tests, significant was adjusted to 0.01 using the Bonferroni method.

\section{Results}

This study was composed of 43 patients (86 sides) who were randomized into an experimental group of 21 patients (42 sides) (14 females and 7 males, with a mean and standard deviation age of $26.1 \pm 4.5$ years) and a control group of 22 patients (44 sides) (16 females and 6 males, with a mean age of 25.4 \pm 2.7 years; range, 18-33 years). Mean mandibular setback extent was $4.00 \pm 1.45 \mathrm{~mm}$ and $4.59 \pm 0.91 \mathrm{~mm}$ (range,

Table 1. Descriptive statistics for control and treatment groups and the results of the independent-samples t-test $(\alpha=0.01)$

\begin{tabular}{|c|c|c|c|c|c|c|}
\hline Time & Group & Mean \pm SD & $95 \% \mathrm{CI}$ & Min & Max & $P$-value \\
\hline \multirow[t]{2}{*}{7 days } & Control & $1.57 \pm 0.50$ & $1.42-1.72$ & 1 & 2 & 0.269 \\
\hline & Experimental & $1.69 \pm 0.52$ & $1.53-1.85$ & 1 & 3 & \\
\hline \multirow[t]{2}{*}{$1 \mathrm{mo}$} & Control & $2.61 \pm 0.54$ & $2.45-2.78$ & 2 & 4 & $0.005 *$ \\
\hline & Experimental & $3.00 \pm 0.70$ & $2.78-3.22$ & 1 & 4 & \\
\hline \multirow[t]{2}{*}{$3 \mathrm{mo}$} & Control & $3.34 \pm 0.57$ & $3.17-3.51$ & 2 & 4 & 0.060 \\
\hline & Experimental & $3.60 \pm 0.66$ & $3.39-3.80$ & 2 & 5 & \\
\hline \multirow[t]{2}{*}{$6 \mathrm{mo}$} & Control & $3.73 \pm 0.59$ & $3.55-3.91$ & 2 & 5 & $0.001 *$ \\
\hline & Experimental & $4.19 \pm 0.67$ & $3.98-4.40$ & 3 & 5 & \\
\hline \multirow[t]{2}{*}{$12 \mathrm{mo}$} & Control & $4.20 \pm 0.76$ & $3.97-4.44$ & 3 & 5 & 0.077 \\
\hline & Experimental & $4.48 \pm 0.63$ & $4.28-4.67$ & 3 & 5 & \\
\hline
\end{tabular}

(SD: standard deviation, CI: confidence interval, Min: minimum, Max: maximum)

$* P<0.01$

Ali Hassani et al: Preoperative imaging of the inferior alveolar nerve canal by cone-beam computed tomography and 1-year neurosensory recovery following mandibular setback through bilateral sagittal split ramus osteotomy: a randomized clinical trial. JKorean Assoc Oral Maxillofac Surg 2020

Table 2. Descriptive statistics for left and right sides and the results of the paired t-test $(\alpha=0.01)$

\begin{tabular}{|c|c|c|c|c|c|c|}
\hline Time & Side & Mean \pm SD & $95 \% \mathrm{CI}$ & Min & Max & $P$-value \\
\hline \multirow[t]{3}{*}{7 days } & Left & $1.53 \pm 0.50$ & $1.38-1.69$ & 1 & 2 & $0.0097 *$ \\
\hline & Right & $1.72 \pm 0.50$ & $1.57-1.88$ & 1 & 3 & \\
\hline & Both & $1.63 \pm 0.51$ & $1.52-1.74$ & 1 & 3 & \\
\hline \multirow[t]{3}{*}{$1 \mathrm{mo}$} & Left & $2.72 \pm 0.59$ & $2.54-2.90$ & 2 & 4 & 0.1971 \\
\hline & Right & $2.88 \pm 0.70$ & $2.67-3.10$ & 1 & 4 & \\
\hline & Both & $2.80 \pm 0.65$ & $2.66-2.94$ & 1 & 4 & \\
\hline \multirow[t]{3}{*}{$3 \mathrm{mo}$} & Left & $3.30 \pm 0.51$ & $3.14-3.46$ & 2 & 4 & 0.0178 \\
\hline & Right & $3.63 \pm 0.69$ & $3.42-3.84$ & 2 & 5 & \\
\hline & Both & $3.47 \pm 0.63$ & $3.33-3.60$ & 2 & 5 & \\
\hline \multirow[t]{3}{*}{$6 \mathrm{mo}$} & Left & $3.77 \pm 0.68$ & $3.56-3.98$ & 2 & 5 & $0.0097 *$ \\
\hline & Right & $4.14 \pm 0.60$ & $3.95-4.32$ & 3 & 5 & \\
\hline & Both & $3.95 \pm 0.67$ & $3.81-4.10$ & 2 & 5 & \\
\hline \multirow[t]{3}{*}{$12 \mathrm{mo}$} & Left & $4.12 \pm 0.70$ & $3.90-4.33$ & 3 & 5 & 0.0063 * \\
\hline & Right & $4.56 \pm 0.67$ & $4.35-4.76$ & 3 & 5 & \\
\hline & Both & $4.34 \pm 0.71$ & $4.18-4.49$ & 3 & 5 & \\
\hline
\end{tabular}

(SD: standard deviation, CI: confidence interval, Min: minimum, Max: maximum)

$* P<0.01$.

Ali Hassani et al: Preoperative imaging of the inferior alveolar nerve canal by cone-beam computed tomography and 1-year neurosensory recovery following mandibular setback through bilateral sagittal split ramus osteotomy: a randomized clinical trial. JKorean Assoc Oral Maxillofac Surg 2020 
1-7 mm) in the experimental and control groups, respectively. There was no significant difference between groups according to sex (Fisher exact test, $P=0.747$ ), age (unpaired t-test, $P=0.492$ ), or amount of movement (unpaired t-test, $P=0.115$ ). No adverse effects were identified.

There were significant differences between the control and experimental neurosensory scores measured 1 month and 6 months after surgery.(Table 1) There were significant differences between the right and left sides during the first, fourth, and last intervals.(Table 2)

Except during the first interval ( 7 days after surgery, Spearman rho $=0.635, P<0.001)$, there were no significant correlations between neurosensory functions perceived by patients on their left and right sides at the second ( $\mathrm{rho}=0.200$, $P=0.199$ ), third (rho $=-0.058, P=0.710$ ), fourth (rho=0.039, $P=0.805)$, or fifth (rho $=0.129, P=0.409)$ follow-up visit. The extent of displacement was not associated significantly with sensory function rate on left or right side for any of the five intervals (all rho values ranged between -0.221 and 0.179 , all $P$-values $>0.250)$.

The mixed-effects model $(\mathrm{n}=86)$ indicated significant effects for jaw side $(P=0.003)$ and CBCT intervention $(P=0.002$; Fig. 2) but not age $(P=0.617)$ or displacement extent $(P=0.122)$.

On the left side $(n=43)$, repeated-measures ANOVA detected significant effects for time $(P=0.001)$ and CBCT intervention $(P=0.005)$. On the right side $(n=43)$, a significant effect was observed for time $(P<0.001)$, while the effect of the intervention was marginally significant $(P=0.066)$. The effect of mandibular displacement was not significant on either side (both $P$-values $>0.280$ ).

\section{Discussion}

We found that performing BSSRO surgery while taking advantage of CBCT information can aid clinicians seeking to reduce neurosensory deficits more rapidly. Only one previous preliminary study by Aizenbud et al. ${ }^{2}$ had compared CBCT and routine imaging versus routine imaging alone before BSSRO surgery. In that study, there was a significant difference between two groups in terms of neurosensory function during the first postoperative year, and they observed positive effects of CBCT imaging on postoperative neurosensory function ${ }^{2}$. However, they did not detect differences between sides $^{2}$. CBCT allowed the surgeon in the present study to observe data that are not available with panoramic imaging alone, such as the distance of the mandibular foramen from the sigmoid notch on the medial side of the ramus, which allowed for better preservation of the nerve bundle. We also increased the number of patients in our sample for better statistical analysis.

Our findings can be discussed considering more general research. Yamamoto et al. ${ }^{17}$ evaluated neurosensory disturbances after BSSRO in patients who had undergone preoperative transaxial CT scans of the IAN canal before surgery and evaluated postoperative neurosensory function with two-point discrimination, light touch discrimination, and a thermocryesthesiometer during the first year after surgery.
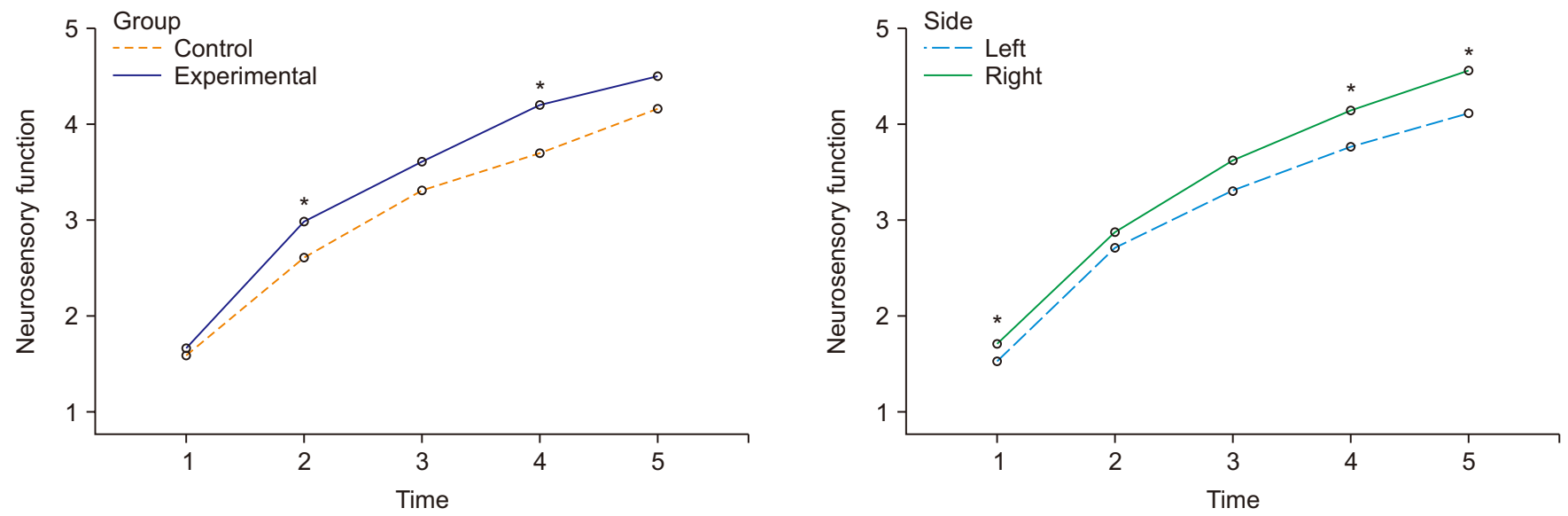

Fig. 2. Marginal means of neurosensory function in the control/experimental groups (left panel) and jaw sides (right panel), estimated at an age of 25.7 years and at a displacement extent of $4.3 \mathrm{~mm}$. The time points 1 to 5 indicate 7 days, 1 month, 3 months, 6 months, and 12 months after surgery, respectively. Significant comparisons $\left({ }^{\star} P<0.01\right)$ between control versus experimental groups (left panel) and between left versus right sides (right panel).

Ali Hassani et al: Preoperative imaging of the inferior alveolar nerve canal by cone-beam computed tomography and 1-year neurosensory recovery following mandibular setback through bilateral sagittal split ramus osteotomy: a randomized clinical trial. J Korean Assoc Oral Maxillofac Surg 2020 
They concluded that neurosensory dysfunction occurred for all cases in which the mandibular canal was in contact with the lateral cortical plate of the mandible, and that a marrow space $0.8 \mathrm{~mm}$ or less between the mandibular canal and external cortical bone was more likely to associate with neurosensory disturbance. Two of the most common causes of injury to the IAN during BSSRO are the variable anatomy of the canal and the relationship of the IAN to the external cortex of the mandible ${ }^{18,19}$. Injury to the nerve bundle most commonly occurs during vertical buccal osteotomy, splitting of segments, screw placement or compression by rigid fixation or during dissection, and osteotomy of the medial side of the ramus ${ }^{5,720,21}$. The IAN canal has a S-shaped course in the ramus and mandibular body. From its entrance to the mandibular foramen, it is close to the lingual cortex; in the region of the third molar, it is most lateral and closest to the buccal cortex; and in the first molar region, it approaches the lingual cortex and finally exits the mental foramen near the premolar region ${ }^{22-24}$. In our study, we obtained CBCT data in the experimental group that allowed us to determine the exact anatomic position of the nerve bundle and secure it from injury during medial dissection, osteotomy, vertical buccal osteotomy, and splitting of segments. This allowed clinicians to minimize neurosensory dysfunction after BSSRO. The reason neurosensory function on the right side improved more rapidly than that on the left side might be due to better access by the surgeon to the right side, resulting in better handling of the tissues and less traction-induced damage. The only other study that has made this comparison found no significant differences between sides ${ }^{2}$, possibly due to small sample size or greater caution exercised during surgery. Future studies are needed for further evaluation of the effect of side, since it might differ from case to case depending on the dominant hand of the surgeon or other operational habits.

Neurosensory disturbances could be attributed to age ${ }^{4}$. In this study, we did not observe a link between age and neurosensory dysfunction despite a wide range of ages among our subjects (and age range might matter ${ }^{1}$ ), but this may reflect the relative youth of our patients rather than an actual lack of association. We did not find the extent of mandibular displacement to be associated with neurosensory deficits, which might be attributed to the low variations in displacement.

This study was limited by some factors. We did not use objective methods of neurosensory evaluation such as blink reflex, electroneurography, or other neurophysiological techniques in our analysis. It remains unclear whether objective or subjective evaluations are superior. A systematic review showed that the incidence of nerve dysfunction is higher after subjective evaluations than objective ones. After one year of evaluation, objective methods determined an incidence of neurosensory dysfunction of $12.8 \%$, while subjective methods yielded an incidence of $18.4 \%{ }^{25}$. Other studies also showed that subjective evaluations of neurosensory dysfunction were associated with greater frequency of detection of sensory impairment than objective methods ${ }^{5,26,27}$. One study found that the results of subjective evaluations of neurosensory function were comparable to the results of two-point discrimination $^{28}$. The most important evaluation factor is patient satisfaction, and good results of aesthetic surgery may be negatively affected by a neurosensory dysfunction. Therefore, we applied subjective evaluations of neurosensory function in the present study. Injury to the IAN can be avoided by applying surgical techniques such as intraoral vertical ramus osteotomy, which yields an incidence of neurosensory dysfunction that is lower than that of BSSRO but has limitations in cases of mandibular deficiencies that require advancement and does not allow rigid fixation that requires a period of maxillomandibular fixation ${ }^{26,29,30}$. The use of the Lindemann bur might be traumatic, especially when the site of surgery is very close to the nerve ${ }^{31}$. However, this was a routine protocol and is also a limitation of many other high- and (especially) low-speed rotating instruments. Moreover, since the protocol was standardized for both groups, it could not affect study outcomes. Finally, the method of randomization used in this study was nonstandard, and we did not use more popular methods such as a random number table.

\section{Conclusion}

In this study, we found that CBCT imaging can allow a surgeon to perform more conservative procedures and therefore indirectly contributes to acceleration of improvement in neurosensory functions over a 1-year period after treatment. We also found that neurosensory disturbances following BSSRO were ameliorated faster on the right side, perhaps due to surgical convenience when operating on that side.

\section{ORCID}

Ali Hassani, https://orcid.org/0000-0001-6159-5691

Vahid Rakhshan, https://orcid.org/0000-0002-9503-3133

Mohammad Hassani, https://orcid.org/0000-0003-2198-3563

Hamidreza Mahaseni Aghdam, https://orcid.org/0000-0002-

$9811-4254$ 


\section{Authors' Contributions}

A.H. conceived the idea and did the surgeries. V.R. analyzed the data and drafted the article. M.H. helped with research procedures. H.M.A. did the surgeries, collected the data, and performed the research.

\section{Ethics Approval and Consent to Participate}

The protocol ethics were approved by the Islamic Azad University's Board of Ethics and Research according to the Helsinki declaration (No. 08-21-214), and informed consent was obtained from each patient.

\section{Conflict of Interest}

No potential conflict of interest relevant to this article was reported.

\section{References}

1. Antony PG, Sebastian A, Varghese KG, Sobhana CR, Mohan S, Soumithran CS, et al. Neurosensory evaluation of inferior alveolar nerve after bilateral sagittal split ramus osteotomy of mandible. $\mathrm{J}$ Oral Biol Craniofac Res 2017;7:81-8.

2. Aizenbud D, Ciceu C, Hazan-Molina H, Abu-El-Naaj I. Relationship between inferior alveolar nerve imaging and neurosensory impairment following bilateral sagittal split osteotomy in skeletal class III cases with mandibular prognathism. Int J Oral Maxillofac Surg 2012;41:461-8.

3. Tamás F. Position of the mandibular canal. Int J Oral Maxillofac Surg 1987;16:65-9.

4. Ylikontiola L, Kinnunen J, Oikarinen K. Factors affecting neurosensory disturbance after mandibular bilateral sagittal split osteotomy. J Oral Maxillofac Surg 2000;58:1234-9; discussion 123940.

5. Brusati R, Fiamminghi L, Sesenna E, Gazzotti A. Functional disturbances of the inferior alveolar nerve after sagittal osteotomy of the mandibular ramus: operating technique for prevention. J Maxillofac Surg 1981;9:123-5.

6. Ylikontiola L, Moberg K, Huumonen S, Soikkonen K, Oikarinen $\mathrm{K}$. Comparison of three radiographic methods used to locate the mandibular canal in the buccolingual direction before bilateral sagittal split osteotomy. Oral Surg Oral Med Oral Pathol Oral Radiol Endod 2002;93:736-42.

7. Westermark A, Bystedt $\mathrm{H}$, von Konow L. Inferior alveolar nerve function after mandibular osteotomies. Br J Oral Maxillofac Surg 1998;36:425-8.

8. Hasani A, Ahmadi Moshtaghin F, Roohi P, Rakhshan V. Diagnostic value of cone beam computed tomography and panoramic radiography in predicting mandibular nerve exposure during third molar surgery. Int J Oral Maxillofac Surg 2017;46:230-5.

9. Neves FS, Souza TC, Almeida SM, Haiter-Neto F, Freitas DQ, Bóscolo FN. Correlation of panoramic radiography and cone beam CT findings in the assessment of the relationship between impacted mandibular third molars and the mandibular canal. Dentomaxillofac Radiol 2012;41:553-7.
10. Arora A, Patil BA, Sodhi A. Validity of the vertical tube-shift method in determining the relationship between the mandibular third molar roots and the inferior alveolar nerve canal. J Korean Assoc Oral Maxillofac Surg 2015;41:66-73.

11. Szalma J, Lempel E, Jeges S, Szabó G, Olasz L. The prognostic value of panoramic radiography of inferior alveolar nerve damage after mandibular third molar removal: retrospective study of 400 cases. Oral Surg Oral Med Oral Pathol Oral Radiol Endod 2010;109:294-302.

12. Kositbowornchai S, Densiri-aksorn W, Piumthanaroj P. Ability of two radiographic methods to identify the closeness between the mandibular third molar root and the inferior alveolar canal: a pilot study. Dentomaxillofac Radiol 2010;39:79-84.

13. Tantanapornkul W, Okochi K, Bhakdinaronk A, Ohbayashi N, Kurabayashi T. Correlation of darkening of impacted mandibular third molar root on digital panoramic images with cone beam computed tomography findings. Dentomaxillofac Radiol 2009;38:11-6.

14. Nakamori K, Tomihara K, Noguchi M. Clinical significance of computed tomography assessment for third molar surgery. World $\mathrm{J}$ Radiol 2014;6:417-23.

15. Obwegeser HL. Orthognathic surgery and a tale of how three procedures came to be: a letter to the next generations of surgeons. Clin Plast Surg 2007;34:331-55.

16. Westermark A, Bystedt H, von Konow L. Patients' evaluation of the final result of sagittal split osteotomy: is it influenced by impaired sensitivity of the lower lip and chin? Int J Adult Orthodon Orthognath Surg 1999;14:135-9.

17. Yamamoto R, Nakamura A, Ohno K, Michi KI. Relationship of the mandibular canal to the lateral cortex of the mandibular ramus as a factor in the development of neurosensory disturbance after bilateral sagittal split osteotomy. J Oral Maxillofac Surg 2002;60:490-5.

18. Rajchel J, Ellis E 3rd, Fonseca RJ. The anatomical location of the mandibular canal: its relationship to the sagittal ramus osteotomy. Int J Adult Orthodon Orthognath Surg 1986;1:37-47.

19. Yoshioka I, Tanaka T, Khanal A, Habu M, Kito S, Kodama M, et al. Relationship between inferior alveolar nerve canal position at mandibular second molar in patients with prognathism and possible occurrence of neurosensory disturbance after sagittal split ramus osteotomy. J Oral Maxillofac Surg 2010;68:3022-7.

20. De Vos W, Casselman J, Swennen GR. Cone-beam computerized tomography (CBCT) imaging of the oral and maxillofacial region: a systematic review of the literature. Int J Oral Maxillofac Surg 2009;38:609-25.

21. Hashiba Y, Ueki K, Marukawa K, Shimada M, Yoshida K, Shimizu $\mathrm{C}$, et al. A comparison of lower lip hypoesthesia measured by trigeminal somatosensory-evoked potential between different types of mandibular osteotomies and fixation. Oral Surg Oral Med Oral Pathol Oral Radiol Endod 2007;104:177-85.

22. Anderson LC, Kosinski TF, Mentag PJ. A review of the intraosseous course of the nerves of the mandible. J Oral Implantol 1991;17:394-403.

23. Gowgiel JM. The position and course of the mandibular canal. J Oral Implantol 1992;18:383-5.

24. Ozturk A, Potluri A, Vieira AR. Position and course of the mandibular canal in skulls. Oral Surg Oral Med Oral Pathol Oral Radiol 2012;113:453-8.

25. Colella G, Cannavale R, Vicidomini A, Lanza A. Neurosensory disturbance of the inferior alveolar nerve after bilateral sagittal split osteotomy: a systematic review. J Oral Maxillofac Surg 2007;65:1707-15.

26. Al-Bishri A, Barghash Z, Rosenquist J, Sunzel B. Neurosensory disturbance after sagittal split and intraoral vertical ramus osteotomy: as reported in questionnaires and patients' records. Int J Oral Maxillofac Surg 2005;34:247-51.

27. Cunningham LL, Tiner BD, Clark GM, Bays RA, Keeling SD, Rugh JD. A comparison of questionnaire versus monofilament assessment of neurosensory deficit. J Oral Maxillofac Surg 
1996;54:454-9; discussion 459-60.

28. Chen N, Neal CE, Lingenbrink P, Bloomquist D, Kiyak HA. Neurosensory changes following orthognathic surgery. Int J Adult Orthodon Orthognath Surg 1999;14:259-67.

29. Bell WH. Modern practice in orthognathic and reconstructive surgery. Philadelphia: Saunders; 1992.

30. Manor Y, Blinder D, Taicher S. Sequence of treatment in mandibular prognathism patients. Cranio 2006;24:95-7.

31. Romeo U, Del Vecchio A, Palaia G, Tenore G, Visca P, Maggiore C. Bone damage induced by different cutting instruments--an in vitro study. Braz Dent J 2009;20:162-8.
How to cite this article: Hassani A, Rakhshan V, Hassani M, Mahaseni Aghdam H. Preoperative imaging of the inferior alveolar nerve canal by cone-beam computed tomography and 1-year neurosensory recovery following mandibular setback through bilateral sagittal split ramus osteotomy: a randomized clinical trial.

J Korean Assoc Oral Maxillofac Surg 2020;46:41-48. https://doi. org/10.5125/jkaoms.2020.46.1.41 